\title{
Research on Methods of Features Extraction for Identifying False RMB Ultraviolet Images
}

\author{
Dan Yin, Ying Zhang, Dahua Xiong, Qinghong Wu \\ University of Science and Technology Liaoning \\ Anshan, China \\ 983206000@qq.com
}

\begin{abstract}
In order to solve the problem of feature selection for the identification of RMB Ultraviolet Images, a method of texture feature extraction image based on Gabor transformation is presented. As we all know, the texture intensity of true currency in each direction is more obvious then counterfeit notes and the difference is more prominent. In this paper, the Gabor transform is carried out in 8 directions. In other words, 8 texture features from RMB are extracted. In order to effectively carry out the false, we gave a full analysis on the image we did. Algorithm proposed in this paper is verified on feature selection of RMB. Furthermore, experimental results show that using this method can intensity currency accurately. This technology has certain utility value and it will be widely used in daily life.
\end{abstract}

Keywords-Ultraviolet image; Features extraction; Gabor transformation; Texture feature;

\section{INTRODUCTION}

At present, the country's cash is a high frequency of circulation, from a small supermarket to the commercial banks are required to identify counterfeit currency. Relying on artificial to carry out the false will bring many disadvantages, such as labor intensity, a long time, the health, the high error rate . Therefore, automatic detection of the paper currency is of great important.

The traditional method of currency identification is mainly used in the transmission of the infrared light and the magnetic signal detection technology. The different printing ink between true currency and false coin will cause the different performance of infrared transmission. Using infrared light to illuminate the RMB and then put a photoelectric conversion device on the back, we can be able to carry out false through the detection of the electrical signal .However, different denomination and different regions of the same currency though security line then process magnetic ink technology, will show different signal waveforms. Magnetic induction electromotive force can be generated by magnetic sensor in the surface cutting magnetic line ${ }^{[1-2]}$. And then we can achieve the purpose of detecting the false by probing the waveform of magnetic signal.

The traditional detection method has the advantages of low cost, signal stability, fast detection, and so on. But with the emerging technology and the characteristics of the detection method has a certain degree of randomness, leading to the above features are easy to be counterfeit. In addition, detect each feature is required to increase the additional hardware, so

This research reported herein was supported by enterprises and institutions entrusted science and technology project No. 2015-10-83. it is difficult to carry out a variety of security features realtime fusion of accurate calculation. In summary, the traditional method can't meet the needs of the current market.

RMB has been added colorless fluorescent ink at the process of printing. Miraculously, the pattern of magnetic ink can't be seen under visible light irradiation, but in the ultraviolet light can be clearly visible. In order to carry out a quick and effective method for the identification of the RMB, this paper analyzes the pattern of the RMB under the ultraviolet irradiation, and studies the method of how to extract the effective features. The experimental results show that the detect method based on the Gabor transform of texture feature greatly improve the effectiveness of the paper currency.

\section{FEATURE SELECTION OF ULTRAVIOLET IMAGE OF NOTE}

In order to improve the security characteristics of the 2005 version of the RMB, fluorescent fiber is added to the pulp of printing RMB in our country. These fluorescent fibers under visible light shows the color reaction is the same with ordinary cotton fiber do, but at a wavelength of $365 \mathrm{~nm}$ ultraviolet irradiation, the atoms of fluorescent substance will be excited by ultraviolet light to rise to an unstable energy level. When the atom returns to a stable level, it will release energy accompanied by a strong yellow and blue fluorescence. It's different from blue and white fluorescence of false currency $^{[3]}$. For example of the fifth edition of 100 yuan denomination, the visible light diagram of true and false currency in the central wavelength of $365 \mathrm{~nm}$ under ultraviolet light is shown in figure 1 and figure 2.

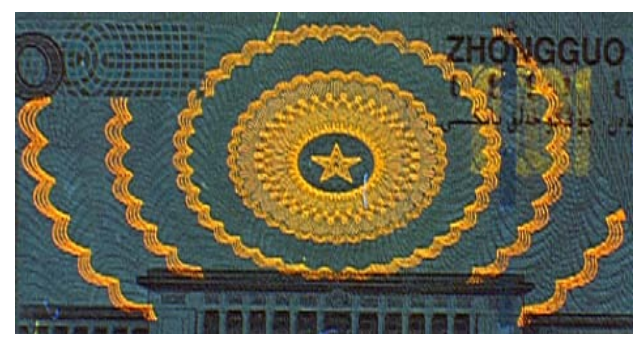

Fig. 1. ultraviolet image of true currency 


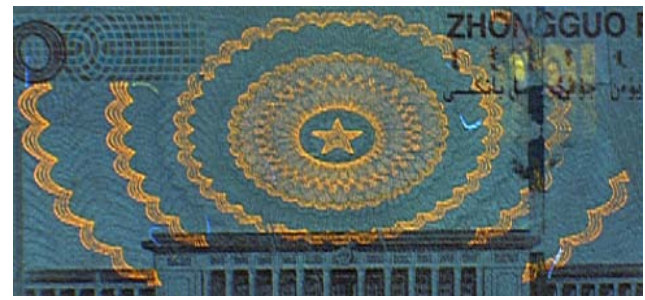

Fig. 2. ultraviolet image of false currency

It's obviously to see the difference between two pictures . The yellow bright ring of true currency is brighter than the false. At the same time, the boundaries of the background pattern of true currency are really clear, instead of ambiguity of the false. Describing ultraviolet image feature areas of true currency and false coin, we can see them in the region of the gray values and images contain texture features are quite different $^{[4-5]}$. So we use the Gabor transform to select the texture features of ultraviolet image.

\section{FEATURE SELECTION BASED ON GABOR FILTER}

The method of digital image processing is divided into two parts: spatial analysis method and frequency domain analysis method. The application of frequency domain analysis is very extensive, such as image enhancement, image restoration, image compression, feature encoding compression and so on. The most famous of it is the Fourier transform ${ }^{[6]}$, nevertheless, it is also have some obvious defects. The classical Fourier transform can only reflect the overall features of the signal (time domain, frequency domain). A frequency in the Fourier spectrum, which is unable to know when to generate. On the other hand, can also be seen from the definition that signals in the entire time domain integral. Therefore, it is reflected by the statistical features of the signal frequency, and the function of local analysis can't actualize. In addition, the signal needs to meet the stationary condition. With the deepening of studies, the Gabor transform and wavelet transform are generated, in order to solve the limitation of the Fourier transform. This paper uses Gabor transform and just prominent it to select the texture features of ultraviolet image.

Gabor transform has many excellent characteristics, Gabor wavelet can not only obtain the minimum uncertainty of time and frequency domain simultaneously, but also very similar to the simple cells in the visual system of human ${ }^{[7]}$. It has good characteristics in the extraction of the target's local space and frequency domain information. Although the Gabor wavelet itself does not constitute an orthogonal basis, it can be formed under certain parameters. It is sensitive to the edge of the image and can provide good characteristics of direction and size selection. What is more, it is not sensitive for the light change, so can provide a good adaptability to illumination changes. These above features make the Gabor wavelet is widely used in visual information.

The basic idea of Gabor Transformation: dividing the signal into many small time intervals and then determining the frequency of it by Fourier transform. The concrete method is to add a sliding window to $\mathrm{f}(\mathrm{T})$, and then make the Fourier transform. By using the Gabor function to transfer from one dimension to two dimensional plane, a two dimensional Gabor filter is generated.

In one-dimensional space, the $\mathrm{F}$ is assumed as a specific function. Then the Gabor transform is defined as:

$$
\begin{aligned}
& G_{f}(a, b, \omega)=\int_{-\infty}^{\infty} f(t) g_{a}^{*}(t-b) e^{-i \omega t} d t \\
& g_{a}(t)=\frac{1}{2 \sqrt{\pi a}} \exp \left(-\frac{t^{2}}{4 a}\right)
\end{aligned}
$$

The second one is the Gaussian function which regarded as "window function" there. Among them, the parameter "b" is used for the parallel moving window, in order to cover the whole time domain. Take (2) as a Gaussian function for two reasons: First, the Fourier transform of the Gaussian function is still Gaussian function, which makes the inverse transform is also a window function of localize ${ }^{[8]}$, and it also reflects the localization of the frequency domain. Second ,the Gabor transform is the optimal window of Fourier transform. It just had the real meaning of the time-frequency analysis after the Gabor transform is put forward. In short, it can provide the local information of both time domain and frequency domain.

Extended to two-dimensional plane, the Gabor filter can well describe the local structure information corresponding to the spatial frequency, spatial position and orientation selectivity. The expression of frequency and direction in Gabor filter is close to the expression of human visual system for frequency and direction, and they are often used for texture representation and description. We can get mathematical expression of Gabor filter:

$$
\begin{aligned}
& \mathrm{g}(\mathrm{x}, \mathrm{y})=\mathrm{s}(\mathrm{x}, \mathrm{y}) \times \mathrm{w}(\mathrm{x}, \mathrm{y}) \\
& s(x, y)=\exp \left(i \times 2 \pi\left(u_{0} x+v_{\mathrm{o}} y\right)\right) \\
& w(x, y)=K \times \exp \left(-\pi\left(\left(\left(x-x_{0}\right)_{r} / a\right)^{2}+\left(\left(y-y_{0}\right)_{r} / b\right)^{2}\right)\right) \\
& K=1 / a b
\end{aligned}
$$

After the transformation, the complete expression is:

$$
g(x, y)=\frac{1}{a b} \times \exp \left(-\pi\left(\left(\left(\frac{\left(x-x_{0}\right)_{r}}{a}\right)^{2}+\left(\frac{\left(y-y_{0}\right)_{r}}{b}\right)^{2}\right)\right) \times \exp \left(i \times 2 \pi\left(u_{0} x+v_{0} y\right)\right)\right.
$$

Gabor filter is used to extract the texture features of paper money, in order to reduce the amount of calculation, the Gabor feature extraction is carried out only on the difference of the ultraviolet image. Take one hundred yuan as an example, the area is shown below.

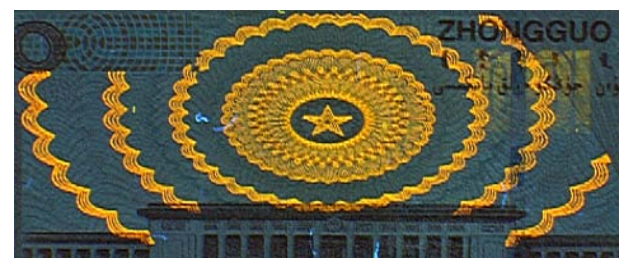

Fig. 3. one hundred yuan feature regional

We have used Gabor filter bank to transform the figure 3 at 8 directions, in other words, 
$\beta=0, \frac{1}{8} \pi, \frac{2}{8} \pi, \frac{3}{8} \pi, \frac{4}{8} \pi, \frac{5}{8} \pi, \frac{6}{8} \pi, \frac{7}{8} \pi$.

The filter bank is shown in the following figures.

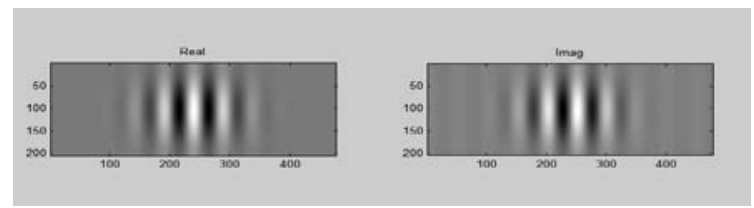

Fig. 4. 0 degree Gabor filter

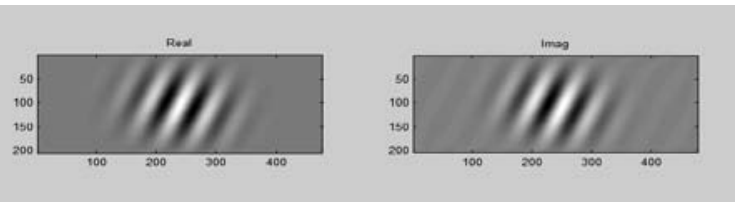

Fig. 5. 22.5 degree Gabor filter

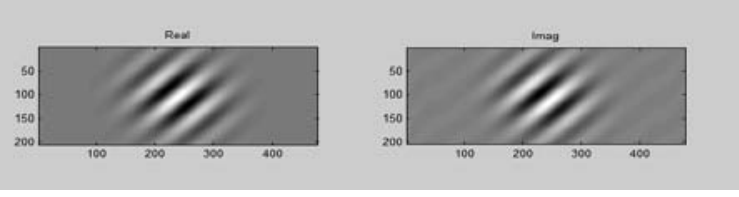

Fig. 6. 45 degree Gabor filter

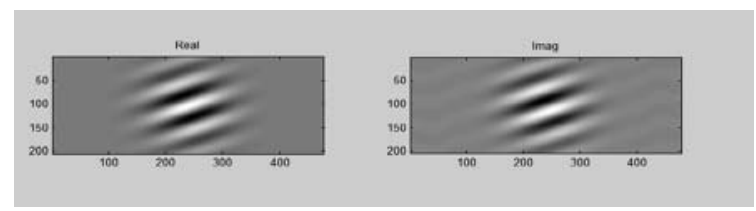

Fig. 7. 67.5 degree Gabor filter

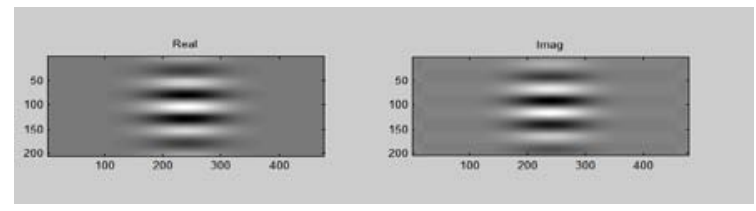

Fig. 8. 90 degree Gabor filter

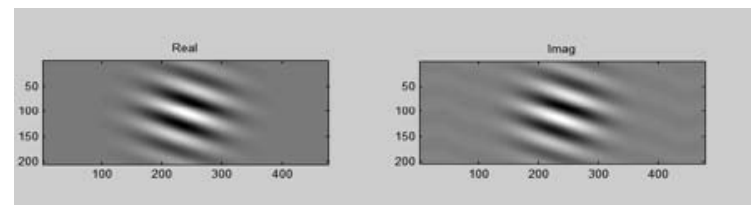

Fig. 9. 112.5 degree Gabor filter

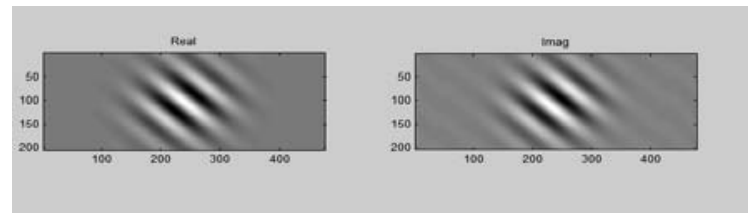

Fig. 10. 135 degree Gabor filter

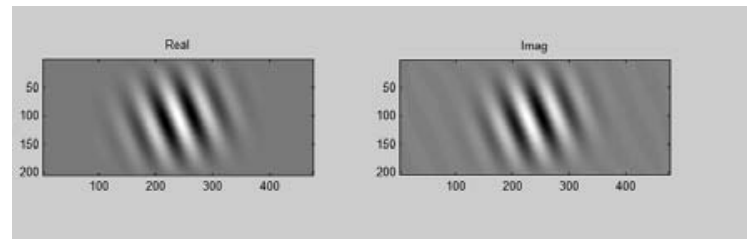

Fig. 11. 157.5 degree Gabor filter

By comparing those figures, we can know the texture feature of true currency is more clear. In this paper, we regard the number of pixels in each direction over a 0.7 times more than the maximum Gabor transform value as a reaction of intensity of texture feature in that direction .

This paper tests one hundred yuan with a number of 100,a half of true and false currency. The average of the maximum value of the list as table I .

TABLE I TRUE AND FALSE CURRENCY GABOR TRANSFORM MAXIMUM AT 8 DIRECTIONS

\begin{tabular}{|c|c|c|c|c|c|c|c|c|}
\hline Direction & $\mathbf{0}$ & $\frac{1}{8} \pi$ & $\frac{2}{8} \pi$ & $\frac{3}{8} \pi$ & $\frac{4}{8} \pi$ & $\frac{5}{8} \pi$ & $\frac{6}{8} \pi$ & $\frac{7}{8} \pi$ \\
\hline True & 5.062 & 4.637 & 5.143 & 3.985 & 4.818 & 3.963 & 5.443 & 5.976 \\
\hline False & 3.543 & 3.246 & 3.600 & 2.789 & 3.373 & 2.774 & 3.810 & 4.183 \\
\hline
\end{tabular}

It can be seen from the table, the average of the maximum value of the true currency is bigger than that of the false.

\section{CONCLUSION AND OUTLOOK}

Under the background of the ultraviolet note image , firstly, the characteristics of the ultraviolet note image are introduced. On the basis of the above, a feature extraction method of true and false currency at eight directions is proposed, which is based on Gabor transform. In the end, the feasibility and reliability of the algorithm are proved by the data.

This paper has solved the problem of feature selection for the identification of RMB ultraviolet images, but still exist some deficiencies. In this paper, the algorithm does not test the RMB which has polluted or incomplete. Therefore, this kind of RMB recognition rate will be biased. I will deep study for the problem to treat this kind of paper money in the near future. 


\section{REFERENCES}

[1] Xiu Qiao Chen, Yi Hua Hu,Jun Mei Li,et al. Background features analysis of infrared image. Infrared Technology, vol.27, no.2, 2005:143146.

[2] BaiJing Qiu, Bao Ling Liu, Chun Du Wu, et al. Applications of nearinfrared image processing in agricultural engineering. Transactions of the CSAE, vol.21, no.5, 2005: 102-106.

[3] Xiu Qiao Chen, Yi Hua Hu,Jun Mei Li,et al.Background features analysis of infrared image.Infrared Technology,vol.27,no.2,2005:143146.

[4] Arash Bahrammirzaee.A comparative survey of artificial intelligence applications in finance: artificial neural networks, expert system and hybrid intelligent systems.Neural Computing and Applications November 2010, Volume 19, Issue 8, pp 1165-1195.

[5] Hiroyuki Onishi,Shoji Tatsumi,A Pattern Defeat Inspection Method by Parallel Grayscale Image Comparison without Precise Image Alignment. IEEE 2006. 14(7):112-116.

[6] Zheng Junli. Signal and system [M]. higher education press.2011.3.

[7] Liu C-Weehsler H.A Gabor feature classifier for face recognition [A].In Proceedings of the Eighth IEEE International Conference on Computer Vision[C],Vancouver,Canada,2001.2:270-275

[8] Chen Xiaolong, Liu Ningbo, Wang Guo. Detection method of sea surface moving target detection based on Gauss's short term fractional Fourier transform[J]. Electronic journal, 2014,42 (5): 971-977. 